\title{
On Ptolemaic metric simplicial complexes
}

\author{
BY S. M. BUCKLEY AND D. J. WRAITH \\ Department of Mathematics, National University of Ireland Maynooth, \\ Maynooth, Co. Kildare, Ireland. \\ e-mail: stephen. buckley@nuim. ie \\ david.wraith@nuim.ie \\ AND J. MCDOUGALL \\ Department of Mathematics and Computer Science, \\ Colorado College, Colorado Springs, Colorado 80903, U.S.A. \\ e-mail: JMcDougall@ColoradoCollege.edu.
}

(Received 10 June 2009; revised 16 December 2009)

\begin{abstract}
We show that under certain mild conditions, a metric simplicial complex which satisfies the Ptolemy inequality is a CAT(0) space. Ptolemy's inequality is closely related to inversions of metric spaces. For a large class of metric simplicial complexes, we characterize those which are isometric to Euclidean space in terms of metric inversions.
\end{abstract}

\section{Introduction}

The aim of this paper is to study some geometric aspects of metric simplicial complexes. (See section 2 for definitions, or the book [BH] for a comprehensive introduction.) Roughly speaking, in a metric simplicial complex the simplexes are all subsets of a fixed Riemannian manifold with constant sectional curvature, and are glued together by isometries. There is a natural way to define a (distance) metric on such an object, and consequently these objects form a large and interesting class of metric spaces.

We are specifically interested in those metric simplicial complexes $(K, d)$ which are Ptolemaic spaces. This means that the following Ptolemy inequality is satisfied for every quadruple of points $x, y, z, p \in(K, d)$ :

$$
d(x, y) d(z, p) \leqslant d(x, z) d(p, y)+d(x, p) d(y, z) .
$$

The significance of this inequality in various metric space settings has been studied recently, for example in [BFW] and [FLS]. Note that a classical result says that Ptolemy's inequality holds in the Euclidean plane, with equality if and only if the points $x, y, z$ and $p$ lie on a circle in that order.

The Ptolemy inequality is closely related to the concept of metric space inversion. Inversion (or reflection) about the Euclidean unit sphere is a bijection on $\mathbb{R}^{n} \backslash\{0\}$, so we can pull back Euclidean distance to get a new distance on $\mathbb{R}^{n} \backslash\{0\}$, namely $i_{0}(x, y):=|x-y| /|x||y|$. Inversion has been generalized to the setting of a metric space $(X, d)$ in $[\mathbf{B H X}]$ : for fixed 
$p \in X$, define

$$
i_{p}(x, y)=\frac{d(x, y)}{d(x, p) d(y, p)}, \quad x, y \in X_{p},
$$

where $X_{p}:=X \backslash\{p\}$. In general this is not a metric on $X_{p}$, but a metric $d_{p}: X_{p} \times X_{p} \rightarrow$ $[0, \infty)$ can be defined which is both subordinate to, and comparable with, $i_{p}$; see [BHX, lemma 3.2].

The definition of $d_{p}$ is more complicated than that of $i_{p}$, so it is natural to ask when $i_{p}$ itself is a metric for all $p \in X$. This reduces to deciding if $i_{p}$ satisfies the triangle inequality, and it is elementary to observe that $\left(X_{p}, i_{p}\right)$ is a metric space for all $p \in X$ if and only if $(X, d)$ is Ptolemaic.

It is not hard to see that CAT(0) spaces are Ptolemaic; see for example [BFW, section 3]. As a corollary, the inversions $i_{p}$ are metrics in every CAT(0) space. On the other hand, the converse statement is not true: there exists a geodesic Ptolemaic space that is not CAT $(0)$; see the comments after [FLS, theorem 1.1].

Nevertheless, if more structure is imposed on the space under consideration, it is possible to give converse statements. Perhaps the earliest result in this direction was due to Schoenberg $[\mathbf{S}]$. This result states that a normed vector space is Ptolemaic if and only if its norm is induced from an inner product (which means the space is Euclidean and hence CAT(0)). More generally, a complete Riemannian or Finsler manifold $M$ is Ptolemaic if and only if it is CAT(0), or equivalently a Hadamard manifold; for a proof, see [BFW] or [K].

The first result in this paper shows that an analogous result is true for metric simplicial complexes.

THEOREM A. Let $K$ be a metric simplicial complex with simplexes of curvature $\kappa$, dimension $n \geqslant 2$ and Shapes $(K)$ finite. If $K$ satisfies the Ptolemy inequality, then $K$ must be $C A T(0)$. In particular we must have $\kappa \leqslant 0$.

The condition 'Shapes $(K)$ finite' above means that the complex contains only finitely many isometry types of simplex.

In [BFW, section 6], it was also shown that inversion can be used to characterize Euclidean space amongst all Riemannian manifolds. In fact if $M$ is a complete Riemannian manifold, then the inversion of $M$ with respect to $p$ has the structure of a Riemannian manifold for all $p \in M$ if and only if $M$ is Euclidean space. Moreover, the inversion is a manifold if and only if it is a length space. Our second main result is an analogue of this result for metric simplicial complexes.

THEOREM B. Let $K$ be a metric simplicial complex with Shapes $(K)$ finite, which is homeomorphic to $\mathbb{R}^{n}$. If for all $p \in K, i_{p}$ is a metric and the inversion of $K$ with respect to $p$ is a length space, then $K$ must be isometric to $\mathbb{R}^{n}$.

Note that some topological assumption such as the condition that $K$ is homeomorphic to $\mathbb{R}^{n}$ is required in Theorem $\mathrm{B}$, since otherwise there are some trivial counterexamples, such as a complex consisting of a single simplex, or certain complexes containing simplexes of differing dimensions.

The remaining sections of this paper are laid out as follows. In section 2 we give all the definitions and background results that we will need. In section 3 we will prove Theorem A and in section 4 we will prove Theorem B. 


\section{Definitions and background results}

We begin this section by recalling some special types of metric space.

We say that $(X, d)$ is a length space if the distance $d(x, y)$ between any pair of points is always equal to the infimum of the lengths of paths between the points. (See [BBI, chapter 2] a full description of the notion of path length in a metric space setting.) A path $\gamma$ of length $d(x, y)$ joining $x, y \in X$ is called a geodesic segment, and is often denoted $[x, y]$. We call $(X, d)$ a geodesic space if all pairs of points can be joined by geodesic segments, that is, the above infimum is always attained.

A geodesic triangle $T(x, y, z)$ is a collection of three points $x, y, z \in X$ together with a choice of geodesic segments $[x, y],[x, z]$ and $[y, z]$. Given such a geodesic triangle $T(x, y, z)$, a comparison triangle will mean a geodesic triangle in a simply-connected constant curvature surface $\bar{T}(\bar{x}, \bar{y}, \bar{z})$, such that corresponding distances coincide: $d(x, y)=$ $\bar{d}(\bar{x}, \bar{y}), d(y, z)=\bar{d}(\bar{y}, \bar{z}), d(z, x)=\bar{d}(\bar{z}, \bar{x})$. (Usually, such a comparison triangle will be in the Euclidean plane.)

Recall the definition of a $\operatorname{CAT}(\kappa)$ space. This is a geodesic metric space $(X, d)$ with the following property. Let $T$ be a geodesic triangle in $X$, and $\bar{T}$ a comparison triangle in constant curvature $\kappa$. Let $D_{\kappa}$ denote the diameter of the unique simply-connected surface of constant curvature $\kappa$ (so $D_{\kappa}=\infty$ if $\kappa \leqslant 0$ ). If the perimeter of $T$ is less than $2 D_{\kappa}$, then given any two points $x, y \in T$ and corresponding comparison points $\bar{x}, \bar{y} \in \bar{T}$ we have

$$
d(x, y) \leqslant \bar{d}(\bar{x}, \bar{y}) .
$$

We say that $T$ is equal to $\bar{T}$ if this inequality is actually an equality for all pairs of points $x, y$.

A metric space is said to be proper if all its closed balls are compact.

LEMMA $2 \cdot 1$. A length space is proper if and only if it is locally compact and complete.

Proof. That locally compact and complete imply proper for length spaces is precisely [BBI, 2.5.22]. In the other direction the local compactness is trivial and the completeness follows from the fact that any Cauchy sequence can be contained in a closed ball.

The one-point extension of $X$ is defined to be

$$
\hat{X}:=\left\{\begin{array}{l}
X \text { when } X \text { is bounded, } \\
X \cup\{\infty\} \text { when } X \text { is unbounded; }
\end{array}\right.
$$

the open sets in $\hat{X}$ include those in $X$ together with complements (in $\hat{X}$ ) of closed balls (in $X$ ). Thus when $X$ is a proper space, $\hat{X}$ is simply its one-point compactification.

Suppose now that $i_{p}$ is actually a metric on $X$ for all $p$. Recall that this is the case if $(X, d)$ is Ptolemaic. Additionally, it is worth noting that $i_{p}$ is a metric if and only if $i_{p}=d_{p}$, where $d_{p}$ is the metric defined in [BHX], mentioned in the introduction.

When $(X, d)$ is unbounded, there is a unique point $p^{\prime}$ in the completion $\left(\hat{X}_{p}, \hat{i}_{p}\right)$ of $\left(X_{p}, i_{p}\right)$ which corresponds to the point $\infty$ in $\hat{X}$. (Any unbounded sequence in $\left(X_{p}, d\right)$ is a Cauchy sequence in $\left(X_{p}, i_{p}\right)$, and any two such sequences are equivalent.) Note that $\hat{i}_{p}\left(x, p^{\prime}\right)=1 / d(x, p)$. We denote this completion $\operatorname{Inv}_{p}(X)$ and refer to it as the inversion of $(X, d)$ with respect to the base point $p$. For example, with this definition, $\operatorname{Inv}_{p}(X)$ will be complete (or proper) whenever $X$ is complete (or proper). 
We now turn our attention to metric simplicial complexes. We follow [BH, p. 98]. Let $M_{\kappa}^{n}$ denote the simply connected $n$-manifold with constant (sectional) curvature $\kappa$. If $n \leqslant m$ then an $n$-plane in $M_{\kappa}^{m}$ will be a subspace isometric to $M_{\kappa}^{n}$. If $(n+1)$ points in $M_{\kappa}^{m}$ do not lie in any $(n-1)$-plane, we say the points are in general position. A geodesic $n$-simplex in $M_{\kappa}^{m}$ is defined to be the convex hull of $(n+1)$ points in general position. If $\kappa>0$ then the vertices (that is, the points defining the convex hull) must lie in an open ball of radius $D_{\kappa} / 2$, with $D_{\kappa}$ as above.

Definition 2.2. Let $\left\{S_{\lambda} \mid \lambda \in \Lambda\right\}$ be a collection of geodesic simplexes with $S_{\lambda} \subset M_{\kappa}^{n_{\lambda}}$. Let $X=\bigcup_{\lambda \in \Lambda}\left(S_{\lambda} \times\{\lambda\}\right)$, let $\sim$ be an equivalence relation on $X$ and set $K:=X / \sim$. Let $p: X \rightarrow K$ be the quotient map and set $p_{\lambda}(x):=p(x, \lambda) . K$ is called an $M_{\kappa}$-simplicial complex if the following two conditions are satisfied:

(1) $p_{\lambda}$ is injective for all $\lambda \in \Lambda$;

(2) if $p_{\lambda}\left(S_{\lambda}\right) \cap p_{\lambda^{\prime}}\left(S_{\lambda^{\prime}}\right) \neq \varnothing$ then there is an isometry $h_{\lambda, \lambda^{\prime}}$ from a face $T_{\lambda} \subset S_{\lambda}$ to a face $T_{\lambda^{\prime}} \subset S_{\lambda^{\prime}}$ such that $p(x, \lambda)=p\left(x^{\prime}, \lambda^{\prime}\right)$ if and only if $x^{\prime}=h_{\lambda, \lambda^{\prime}}(x)$.

In this paper, we will always assume that our complexes are connected.

Definition $2 \cdot 3$. The set of isometry classes of the faces of the geodesic simplexes $S_{\lambda}$ will be denoted Shapes $(K)$.

An $M_{\kappa}$-complex $K$ becomes a pseudometric space when equipped with the following natural metric. Given any two points $x$ and $y$ in $K$, consider a sequence of points $x=$ $x_{1}, x_{2}, \ldots, x_{n}=y$ with the property that every adjacent pair of points belong to a common simplex. As a result, the distance between any two adjacent points can be found, and so the 'length' of the sequence can be computed. The pseudometric $d(x, y)$ is then defined to be the infimum of the lengths of all such sequences linking $x$ and $y$. If this pseudometric is actually a metric, we call $K$ a metric simplicial complex.

The following result from Bridson's thesis (see [BH, p. 97]) shows that metric simplicial complexes have good properties:

THEOREM 2.4. An $M_{\kappa}$-simplicial complex $K$ with Shapes $(K)$ finite is a metric simplicial complex, and moreover it is a complete geodesic space.

With a view towards exploring some of the more detailed structure of metric simplicial complexes, we introduce the concept of a $\kappa$-cone over a metric space [BH, p. 59]. Given $\kappa \in \mathbb{R}$ and a metric space $(X, d)$, the $\kappa$-cone over $X, C_{\kappa} X$ is given by $X \times[0, \infty) / \sim$ if $\kappa \leqslant 0$ and $X \times\left[0, D_{\kappa} / 2\right] / \sim$ in the case $\kappa>0$, where in either case $(t, x) \sim\left(t^{\prime}, x^{\prime}\right)$ if and only if $t=t^{\prime}=0$ or we have equality of pairs. The equivalence class corresponding to the point 0 is the vertex of the cone. We define a metric $d_{C}$ on $C_{\kappa} X$ as follows. Let $p=[x, t]$ and $p^{\prime}=\left[x^{\prime}, t^{\prime}\right]$.

If $\kappa=0$ then set

$$
d_{C}\left(p, p^{\prime}\right)=t^{2}+t^{\prime 2}-2 t t^{\prime} \cos \left(\min \left\{\pi, d\left(x, x^{\prime}\right)\right\}\right) .
$$

If $\kappa<0$ then define $d_{C}$ via

$$
\begin{aligned}
& \cosh (\sqrt{-\kappa}) d_{C}\left(p, p^{\prime}\right) \\
& \quad=\cosh (\sqrt{-\kappa} t) \cosh \left(\sqrt{-\kappa} t^{\prime}\right)-\sinh (\sqrt{-\kappa} t) \sinh \left(\sqrt{-\kappa} t^{\prime}\right) \cos \left(\min \left\{\pi, d\left(x, x^{\prime}\right)\right\}\right) .
\end{aligned}
$$


If $\kappa>0$ then define $d_{C}$ via

$\cos (\sqrt{\kappa}) d_{C}\left(p, p^{\prime}\right)=\cos (\sqrt{\kappa} t) \cos \left(\sqrt{\kappa} t^{\prime}\right)+\sin (\sqrt{\kappa} t) \sin \left(\sqrt{\kappa} t^{\prime}\right) \cos \left(\min \left\{\pi, d\left(x, x^{\prime}\right)\right\}\right)$.

Definition 2.5. For any point $x$ in a geodesic simplex $S$, the link of $x$ in $S, \operatorname{Lk}(x, S)$ is the set of unit vectors at $x$ which point into $S$. If $x$ in an element of a metric simplicial complex $K$, the link of $x$ in $K, \operatorname{Lk}(x, K)$ is the union of the links of $x$ in all simplexes to which $x$ belongs. (See [BH, pp. 102-103] for an alternative description.)

One can define a natural (angular) pseudometric on $\operatorname{Lk}(x, K)$.

Fact 2.6 [BH, p. 103]. For any $M_{\kappa}$-complex $K$ and any point $x \in K, L k(x, K)$ is an $M_{1}$-simplicial complex. Hence if Shapes $(K)$ is finite, then $L k(x, K)$ is a metric simplicial complex and a complete geodesic space.

Note that an $M_{\kappa}$-complex is not necessarily a $\mathrm{CAT}(\kappa)$ space. In particular, a link complex is not necessarily a CAT(1) space. This motivates the following definition:

Definition 2.6 [BH, p. 103]. An $M_{\kappa}$-simplicial complex $K$ satisfies the link condition if for every vertex $v \in K$, the link complex $\operatorname{Lk}(v, K)$ is a $\operatorname{CAT}(1)$ space.

The following result will be crucial in the proof of Theorem A in section 2. It is a combination of [BH, II.5.4] and [BH, II.5.6].

THEOREM 2.8. Let $K$ be an $M_{\kappa}$-simplicial complex with Shapes $(K)$ finite.

(a) If $\kappa \leqslant 0$, then the following conditions are equivalent:

(i) $K$ is a $C A T(\kappa)$ space;

(ii) $K$ satisfies the link condition and contains no isometrically embedded circles.

(b) If $\kappa>0$, then the following conditions are equivalent:

(i) $K$ is a $C A T(\kappa)$ space;

(ii) $K$ satisfies the link condition and contains no isometrically embedded circles of length less than $2 \pi / \sqrt{\kappa}$.

(c) If $K$ is a two-dimensional complex, then it satisfies the link condition if and only if for each vertex $v \in K$, every injective loop in $L k(v, K)$ has length at least $2 \pi$.

The final result in this section is essentially [BH, I.7.17] in the special case of a complex $K$ with $\operatorname{Shapes}(K)$ finite.

THEOREM 2.9. For an $M_{\kappa}$-simplicial complex $K$ and a point $x \in K$, the $\epsilon$-ball about $x, B(x, \epsilon)$, is isometric to the $\epsilon$-ball about the vertex in $C_{\kappa}(L k(x, K))$ for all $\epsilon$ sufficiently small.

\section{The proof of Theorem $A$}

Proof. First note that the Ptolemaic condition must hold in each simplex of $K$, so as noted in the Introduction this means each simplex must individually be a CAT(0) space. In turn this means that we must have $\kappa \leqslant 0$.

As $K$ is Ptolemaic, it cannot contain an isometrically embedded circle, as the "quarter points' would violate the Ptolemy inequality. Therefore, by Theorem $2 \cdot 8$, we see that $K$ is $\mathrm{CAT}(\kappa)$ if and only if it satisfies the link condition at each vertex. (The Shapes $(K)$ condition is required for this theorem.) By the same result, the link $L k(v, K)$ of some vertex $v$ of $K$ 
is a CAT(1) space if and only if $L k(v, K)$ satisfies the link condition at each of its vertices and contains no isometrically embedded $S^{1}$ of length strictly less than $2 \pi$. We will use the term 'short' to describe a circle of length less than $2 \pi$. Therefore $K$ fails to satisfy the link condition if and only if the link in $L k(v, K)$ of some vertex either fails to satisfy the link condition or $L k(v, K)$ has a short isometrically embedded circle.

If the link condition fails in $\operatorname{Lk}(v, K)$ then either the link condition fails in a link of the link, or some link of the link has a short isometrically embedded $S^{1}$. (From now on we will suppress the vertices from the link notation, so $L k(K)$ will denote the original link, a link of this link will be written $L k^{2}(K)$ and so on.) If the link condition keeps failing in $L k(K)$, $L k^{2}(K), L k^{3}(K), \ldots$ then eventually the link condition will fail in a 1 -complex. But the link condition fails in a 1-complex if and only if there is an isometrically embedded (i.e. injective) short loop. We therefore arrive at the following

Observation. A Ptolemaic $M_{\kappa}$-complex $K$ fails to be a CAT $(\kappa)$ space $(\kappa \leqslant 0)$ if and only if some $L k^{m}(K), m \geqslant 1$, has an isometrically embedded short loop.

Our strategy is to show that the existence of an isometrically embedded $S^{1}$ in some $L k^{m}(K)$ gives a contradiction with the original complex $K$ being Ptolemy. More specifically, the quarter-points in this $S^{1}$ can be associated to four points in $K$ (by the "method of association' below), for which the Ptolemy inequality can be shown to fail. Thus $K$ has to be $\operatorname{CAT}(\kappa)$, and in particular $\operatorname{CAT}(0)$.

Method of Association. To the $S^{1}$ in $L k^{m}(K)$ we actually associate an $S^{1}$ in $K$. The desired four points are then the points in $K$ corresponding to the quarter-points in the original circle.

There is an $\epsilon>0$ such that the $\epsilon$-neighbourhood of the cone point in $C_{1} L k^{m}(K)$ is isometric to the $\epsilon$-neighbourhood of the central vertex for $L k^{m-1}(K)$. (Here, $L k^{0}(K)$ should be interpreted as $K$ itself.) The $S^{1} \subset L k^{m}(K)$ gives a topological circle $\epsilon S^{1} \subset C_{1} L k^{m}(K)$, and in turn this gives a topological circle in $L k^{m-1}(K)$. Continuing in this way (with the same suitably small $\epsilon$ ) we will eventually produce an embedded $S^{1}$ in $K$.

In order to show that the Ptolemy inequality fails for our four points in $K$, we need to investigate how distance alters when we embed points into successive 1-cones, and ultimately into $\kappa$-cones, with $\kappa \leqslant 0$.

Consider two points separated by a distance $D$ in some metric space $Y$. (Assume $D \leqslant$ $\pi$.) Then according to the cone metric definitions given in section 2, the separation of the corresponding points at a distance $\epsilon$ from the vertex in $C_{1} Y$ is

$$
\cos ^{-1}\left(\cos ^{2} \epsilon+\left(\sin ^{2} \epsilon\right) \cos D\right)=\cos ^{-1}\left(1-\left(\sin ^{2} \epsilon\right)(1-\cos D)\right) .
$$

Similarly, the separation of the corresponding points at a distance $\epsilon$ from the vertex in the 'cone over the cone' $C_{1}^{2} Y$ is

$$
\begin{aligned}
& \cos ^{-1}\left(1-\left(\sin ^{2} \epsilon\right)\left[1-\cos \left(\cos ^{-1}\left[\left(1-\sin ^{2} \epsilon\right)(1-\cos D)\right]\right)\right]\right) \\
& \quad=\cos ^{-1}\left(1-\left(\sin ^{2} \epsilon\right)\left[1-\left(1-\left(\sin ^{2} \epsilon\right)(1-\cos D)\right)\right]\right) \\
& \quad=\cos ^{-1}\left(1-\left(\sin ^{4} \epsilon\right)(1-\cos D)\right) .
\end{aligned}
$$

Similarly, the corresponding distance in $C_{1}^{r} Y$ is $\cos ^{-1}\left(1-\left(\sin ^{2 r} \epsilon\right)(1-\cos D)\right)$.

The case $\kappa=0$.

Suppose the points at the above separation are finally embedded at a distance $\epsilon$ from the vertex of a 0 -cone. By the cone metric definitions in section 2 , the separation is 
then

$$
\begin{aligned}
{\left[2 \epsilon^{2}-2 \epsilon^{2} \cos \left(\cos ^{-1}\left[1-\left(\sin ^{2 r} \epsilon\right)(1-\cos D)\right]\right)\right]^{\frac{1}{2}} } \\
\quad=\epsilon \sqrt{2}\left[1-\left(1-\left(\sin ^{2 r} \epsilon\right)(1-\cos D)\right)\right]^{\frac{1}{2}} \\
\quad=\epsilon \sqrt{2}\left(\sin ^{r} \epsilon\right) \sqrt{1-\cos D .}
\end{aligned}
$$

In the original isometrically embedded $S^{1}$, suppose the separation of adjacent quarter points is $D<\pi / 2$. The separation of opposite points is then $2 D$.

We check Ptolemy's inequality for the corresponding points in $K$. Using $(*)$ we see that Ptolemy will fail if

$$
2 \epsilon^{2}\left(\sin ^{2 r} \epsilon\right)(1-\cos 2 D)>4 \epsilon^{2}\left(\sin ^{2 r} \epsilon\right)(1-\cos D) ;
$$

that is, if

$$
1-\cos 2 D>2(1-\cos D) .
$$

But $\cos 2 D=2 \cos ^{2} D-1$, so this inequality is really

$$
2-2 \cos ^{2} D>2-2 \cos D,
$$

or more simply

$$
\cos D>\cos ^{2} D \text {. }
$$

But $D \in(0, \pi / 2)$, therefore $\cos D \in(0,1)$ and so the inequality must be true.

The case $\kappa<0$.

As in the $\kappa=0$ case, suppose the four points with (adjacent) separation $\cos ^{-1}(1-$ $\left.\left(\sin ^{2 r} \epsilon\right)(1-\cos D)\right)$ are finally embedded at a distance $\epsilon$ from the vertex of a $\kappa$-cone, with $\kappa<0$. By the cone metric definitions in section 2 , the separation is then

$$
\begin{gathered}
\cosh ^{-1}\left(\cosh ^{2} \epsilon-\left(\sinh ^{2} \epsilon\right) \cos \left(\cos ^{-1}\left[1-\left(\sin ^{2 r} \epsilon\right)(1-\cos D)\right]\right)\right) \\
=\cosh ^{-1}\left(\cosh ^{2} \epsilon-\left(\sinh ^{2} \epsilon\right)\left[1-\left(\sin ^{2 r} \epsilon\right)(1-\cos D)\right]\right),
\end{gathered}
$$

and the separation of opposite points is

$$
=\cosh ^{-1}\left(\cosh ^{2} \epsilon-\left(\sinh ^{2} \epsilon\right)\left[1-\left(\sin ^{2 r} \epsilon\right)(1-\cos 2 D)\right]\right) .
$$

We show that for $\epsilon$ sufficiently small, Ptolemy's inequality fails for these distances.

Setting $D^{\prime}=\cos ^{-1}\left(1-\left(\sin ^{2 r} \epsilon\right)(1-\cos D)\right)$ and $D^{\prime \prime}=\cos ^{-1}\left(1-\left(\sin ^{2 r} \epsilon\right)(1-\cos 2 D)\right)$, let us label the corresponding separations $S^{\prime}(\epsilon)$ and $S^{\prime \prime}(\epsilon)$ respectively. It follows from the cone metric definitions that these separations are equal to the length of the third side in an isosceles triangle in the simply connected space of constant curvature $\kappa$, where the equal sides have length $\epsilon$, and the angle between the sides is $D^{\prime}$ respectively $D^{\prime \prime}$.

It will be convenient to find alternative expressions for $S^{\prime}(\epsilon)$ and $S^{\prime \prime}(\epsilon)$ based on the sine law for triangles in hyperbolic space. Note that for a geodesic triangle in the simply connected space of constant curvature $\kappa<0$, the sine law reads

$$
\frac{\sinh a}{\sin A}=\frac{\sinh b}{\sin B}=\frac{\sinh c}{\sin C}
$$

where $a, b, c$ are the side lengths and $A, B, C$ are the angles (see [C, p. 94]).

Consider splitting each isosceles triangle into two equal triangles by introducing a line dividing the angle $D^{\prime}$ (respectively $D^{\prime \prime}$ ) in half. The point at which this line meets the opposite 
side is clearly the point on that side closest to the cone vertex. The angle made between the two lines is therefore $\pi / 2$.

Applying the sine law to one of our 'half-triangles' gives

$$
\frac{1}{\sqrt{-\kappa}} \frac{\sinh (\epsilon \sqrt{-\kappa})}{\sin (\pi / 2)}=\frac{1}{\sqrt{-\kappa}} \frac{\sinh \left(\sqrt{-\kappa} S^{\prime}(\epsilon) / 2\right)}{\sin \left(D^{\prime} / 2\right)},
$$

which after rearranging gives

$$
S^{\prime}(\epsilon)=\frac{2}{\sqrt{-\kappa}} \sinh ^{-1}\left(\sin \left(D^{\prime} / 2\right) \sinh (\epsilon \sqrt{-\kappa})\right) .
$$

Similarly for $S^{\prime \prime}$ and $D^{\prime \prime}$. Expanding this as a Taylor series about $\epsilon=0$ gives

$$
\begin{aligned}
& S^{\prime}(\epsilon)=2 \epsilon \sin \left(D^{\prime} / 2\right)+O\left(\epsilon^{3}\right), \\
& S^{\prime \prime}(\epsilon)=2 \epsilon \sin \left(D^{\prime \prime} / 2\right)+O\left(\epsilon^{3}\right) .
\end{aligned}
$$

The Ptolemy inequality will fail for our chosen points if $S^{\prime \prime 2}(\epsilon)>2 S^{\prime 2}(\epsilon)$, that is, if

$$
4 \epsilon^{2} \sin ^{2}\left(D^{\prime \prime} / 2\right)+O\left(\epsilon^{4}\right)>8 \epsilon^{2} \sin ^{2}\left(D^{\prime} / 2\right)+O\left(\epsilon^{4}\right)
$$

or equivalently

$$
\sin ^{2}\left(D^{\prime \prime} / 2\right)+O\left(\epsilon^{2}\right)>2 \sin \left(D^{\prime} / 2\right)+O\left(\epsilon^{2}\right) .
$$

We claim that this is true for all sufficiently small $\epsilon$. Before we can establish this, however, we need two lemmas.

LEMMA 3·1. For $\lambda>\sqrt{2}$ and $x$ sufficiently small, the following inequality holds:

$$
\sin ^{2} x>2 \sin ^{2}(x / \lambda)
$$

Proof. For $x$ small we have $\sin ^{2} x=x^{2}+O\left(x^{4}\right)$. Therefore

$$
2 \sin ^{2}(x / \lambda)=2(x / \lambda)^{2}+O\left(x^{4}\right) .
$$

For $x$ so small that the $O\left(x^{4}\right)$ terms are irrelevant, establishing the inequality reduces to showing that $x^{2}>2(x / \lambda)^{2}$, which is true since $\lambda>\sqrt{2}$.

Lemma 3.2. Given $D \in(0, \pi / 2)$, choose $\lambda \in(\sqrt{2}, \sqrt{2(1+\cos D)})$. Then for $\epsilon>0$ sufficiently small (depending on $\lambda$ ) we have

$$
\cos ^{-1}(1-\epsilon(1-\cos 2 D))>\lambda \cos ^{-1}(1-\epsilon(1-\cos D)) .
$$

Notice that both sides would be zero if we were allowed to set $\epsilon=0$. We examine the derivatives with respect to $\epsilon$ of each side in the above inequality.

$$
\begin{aligned}
\frac{d}{d \epsilon} \cos ^{-1}(1-\epsilon(1-\cos 2 D)) & =\frac{1-\cos 2 D}{\sqrt{2 \epsilon(1-\cos 2 D)-\epsilon^{2}(1-\cos 2 D)^{2}}} \\
\frac{d}{d \epsilon} \cos ^{-1}(1-\epsilon(1-\cos D)) & =\frac{1-\cos D}{\sqrt{2 \epsilon(1-\cos D)-\epsilon^{2}(1-\cos D)^{2}}} .
\end{aligned}
$$

We can therefore establish the truth of our inequality by showing that for $\epsilon$ sufficiently small:

$$
\frac{1-\cos 2 D}{\sqrt{2 \epsilon(1-\cos 2 D)-\epsilon^{2}(1-\cos 2 D)^{2}}}>\frac{\lambda(1-\cos D)}{\sqrt{2 \epsilon(1-\cos D)-\epsilon^{2}(1-\cos D)^{2}}}
$$


or equivalently

$$
\frac{1-\cos 2 D}{\lambda(1-\cos D)}>\sqrt{\frac{2(1-\cos 2 D)-\epsilon(1-\cos 2 D)^{2}}{2(1-\cos D)-\epsilon(1-\cos D)^{2}}} .
$$

As $\epsilon \rightarrow 0$, the right-hand side tends to

$$
\sqrt{\frac{1-\cos 2 D}{1-\cos D}}
$$

It therefore suffices to show that

$$
\frac{1-\cos 2 D}{\lambda(1-\cos D)}>\sqrt{\frac{1-\cos 2 D}{1-\cos D}}
$$

or equivalently

$$
\frac{1-\cos 2 D}{1-\cos D}>\lambda^{2}
$$

Using the double angle formula for $\cos 2 D$ in the above and rearranging, we obtain

$$
-2 \cos ^{2} D+\lambda^{2} \cos D+\left(2-\lambda^{2}\right)>0 \text {. }
$$

The roots of this quadratic expression are $\cos D=1$ and $\cos D=\left(\lambda^{2}-2\right) / 2$. Therefore the quadratic expression is positive precisely when $\cos D \in\left(\left(\lambda^{2}-2\right) / 2,1\right)$, assuming $\lambda<2$. But $\cos D<1$ anyway since $D \in(0, \pi / 2)$, so we only require $\cos D>\left(\lambda^{2}-2\right) / 2$, i.e. $\lambda<\sqrt{2(1+\cos D)}$, as claimed.

Proof of Theorem A continued.

Now let us return to the inequality (粶). Lemma 3.2 shows that for a suitable choice of $\lambda$, $D^{\prime \prime}>\lambda D^{\prime}$ for $\epsilon$ suitably small. By Lemma $3 \cdot 1$, for this $\lambda$ and $\epsilon$ sufficiently small we have

$$
\sin ^{2}\left(D^{\prime \prime} / 2\right)>\sin ^{2}\left(\lambda D^{\prime} / 2\right)>2 \sin ^{2}\left(\frac{\lambda D^{\prime}}{2 \lambda}\right)=2 \sin ^{2}\left(D^{\prime} / 2\right) .
$$

Thus for $\epsilon$ sufficiently small we see that (粶) holds, and thus the Ptolemy inequality fails.

Finally, note that in both the $\kappa=0$ and $\kappa<0$ cases, we did not consider the situation where the isometrically embedded $S^{1}$ is in $K$, as opposed to some $L k^{m}(K)$. However this situation is trivial, as the failure of the Ptolemy inequality is equivalent to showing $4 D^{2}>$ $2 D^{2}$, which is clearly true.

\section{The proof of Theorem $B$}

Before proving Theorem B we need a sequence of lemmas.

LEMMA 4.1. If $\left(X_{p}, i_{p}\right)$ is a length space, then so is $\operatorname{Inv}_{p}(X)=\left(\hat{X}_{p}, \hat{i}_{p}\right)$.

Proof. It is clear that the only issue is with distances to the point $p^{\prime} \in \operatorname{Inv}_{p}(X)$ corresponding to the point at infinity in the completion of $(X, d)$ (in the case that $(X, d)$ is unbounded).

Consider any $d$-unbounded Cauchy sequence $\left\{y_{i}\right\} \subset \operatorname{Inv}_{p}(X)$. Given any $x \in X$ and $\epsilon>0$, we construct a path from $x$ to $p^{\prime}$ with length strictly less than $\hat{i}_{p}\left(x, p^{\prime}\right)+\epsilon$. We do this as follows. Choose $I_{0}$ such that for all $i, j \geqslant I_{0}$ we have $\hat{i}_{p}\left(y_{i}, y_{j}\right)<\epsilon / 8$. By 
removing points of the sequence and re-labelling if necessary, we can assume without loss of generality that for all $i \geqslant I_{0}$,

$$
\hat{i}_{p}\left(y_{i}, y_{i+1}\right)<\frac{\epsilon}{8} \cdot 2^{-i} .
$$

Moreover, since $\left(X_{p}, i_{p}\right)$ is a length space, we can choose a path from $y_{i}$ to $y_{i+1}$ for each $i \geqslant I_{0}$ with length strictly less than

$$
\hat{i}_{p}\left(y_{i}, y_{i+1}\right)+\frac{\epsilon}{8} \cdot 2^{-i}<\frac{\epsilon}{4} \cdot 2^{-i} .
$$

We can also choose a path from $x$ to $y_{I_{0}}$ of length strictly less than

$$
\hat{i}_{p}\left(x, y_{I_{0}}\right)+\frac{\epsilon}{4} \text {. }
$$

Therefore concatenating this with the paths between the $\left\{y_{i}\right\}, i \geqslant I_{0}$, gives a path of total length at most $\hat{i}_{p}\left(x, y_{I_{0}}\right)+(\epsilon / 2)$. Parameterising this path by arclength and calling it $\gamma(t)$ gives a map

$$
\gamma:[0, L) \longrightarrow \hat{X}_{p}
$$

and clearly

$$
\lim _{t \rightarrow L} \hat{i}_{p}\left(\gamma(t), p^{\prime}\right)=0 .
$$

We can therefore 'complete' the path by adding the point $p^{\prime}$, to get a path $\hat{\gamma}(t)$ defined on the interval $[0, L]$. Obviously, adding this point does not affect the length. We have therefore constructed a path $\hat{\gamma}$ from $x$ to $p^{\prime}$ with length at most $\hat{i}_{p}\left(x, y_{I_{0}}\right)+(\epsilon / 2)$. Using the triangle inequality we see that

$$
\hat{i}_{p}\left(x, y_{I_{0}}\right) \leqslant \hat{i}_{p}\left(x, p^{\prime}\right)+\hat{i}_{p}\left(y_{I_{0}}, p^{\prime}\right) \leqslant \hat{i}_{p}\left(x, p^{\prime}\right)+\frac{\epsilon}{8} .
$$

Therefore the length of $\hat{\gamma}$ satisfies

$$
\text { length of } \hat{\gamma}<\hat{i}_{p}\left(x, p^{\prime}\right)+\frac{\epsilon}{2}+\frac{\epsilon}{8}<\hat{i}_{p}\left(x, p^{\prime}\right)+\epsilon
$$

as claimed.

LEMMA 4.2. Let $K$ be an $M_{\kappa}$-complex with Shapes $(K)$ finite, which is homeomorphic to $\mathbb{R}^{n}$, for which $i_{p}$ is a length metric. Then $\hat{i}_{p}$ is a geodesic metric.

Proof. By Lemma $4 \cdot 1, \hat{i}_{p}$ is also a length metric. Now a complete locally compact length space is a geodesic space (see [BBI, 2.5.23]), and so it suffices to show that $\operatorname{Inv}_{p}(K)$ is complete and locally compact. By Lemma $2 \cdot 1$, this in turn is equivalent to properness. However, as noted in section 2 (or see [BHX, p. 6]), if $(X, d)$ is proper, so is $\operatorname{Inv}_{p}(X)$. Therefore in our case, we will be done if we can show that the complex $(K, d)$ is proper, or equivalently that it is complete and locally compact. As local compactness is preserved by homeomorphism, and $K \cong \mathbb{R}^{n}$, the local compactness of $K$ is clear. Completeness is given by Theorem $2 \cdot 4$.

LeMmA 4.3. Suppose the metric simplicial complex $K$ is such that Shapes $(K)$ is finite, and $K$ is homeomorphic to $\mathbb{R}^{n}, n \geqslant 2$. If every triangle in $K$ is isometric to its comparison triangle in $\mathbb{R}^{2}$, then $K$ is isometric to $\mathbb{R}^{n}$.

Proof. It clearly suffices to show this for a neighbourhood of every vertex in $K$. 
Embed $n+1$ points into $K=K^{n}$ in general position, so that the chosen vertex lies in the convex hull. The hull, $\Delta^{n}$, is homeomorphic to a standard $n$-simplex. We show how to construct an isometry from this region of $K$ to a region of $\mathbb{R}^{n}$. We construct this isometry on successive skeleta of $\Delta^{n}$.

Consider a geodesic triangle forming part of the 1-skeleton of $\Delta^{n}$, and consider a comparison triangle in $\mathbb{R}^{n}$. We automatically have an isometry between these triangles.

Next we 'fill-in' the triangles, that is, extend the isometry across the interior. To do this, consider the one parameter family of geodesics from a fixed vertex $v$ of the triangle in $K$ to each point of the opposite side. The corresponding lines in our Euclidean triangle lead to a one-to-one extension map in the obvious way. We claim that this is actually an isometry.

Given any two points $x, y$, in the interior of the triangle in $K$, let $\bar{x}$ and $\bar{y}$ denote the points where the extension of the geodesics $v x$ respectively $v y$ meet the side of the original triangle opposite to $v$. Our comparison triangle assumption applied to the triangle $v \bar{x} \bar{y}$ means that the length of the side $x \bar{y}$ is the same as the corresponding length in the Euclidean comparison triangle. Now apply the assumption to the triangle $v x \bar{y}$ to show that the length of $x y$ is the same as the Euclidean comparison distance. Thus distances agree under this mapping, as claimed.

If $n=2$, we are done. Otherwise, the original triangle in $K$ is a face of some tetrahedron in $\Delta^{n}$. This tetrahedron has one vertex which is not a vertex of the triangle. Consider another face of the tetrahedron. We can find a comparison triangle in Euclidean space which intersects our original comparison triangle in a common side. Again we can 'fill-in' and extend our mapping to the union of the two triangles.

By pivoting the second Euclidean triangle about the common edge, we can clearly arrange for the distance between the two vertices not on the common edge to be the same as that for the corresponding vertices in $\Delta^{n}$. We then have that all faces of the tetrahedron defined by the points introduced into $\mathbb{R}^{n}$ are comparison triangles for the corresponding faces in $\Delta^{n}$. We can therefore extend our isometry to an isometry of each face.

We claim that this is actually a global isometry of the union of the faces. Given two points in different faces of $\Delta^{n}$, consider the minimal geodesic in $\Delta^{n}$ linking them, and in particular consider the point(s) at which the geodesic switches faces. Joining the corresponding three (or more) points in the Euclidean picture, we obtain a curve of the same length in $\mathbb{R}^{n}$. Suppose this is not a minimal geodesic for our Euclidean tetrahedron: then the pre-image in $\Delta^{n}$ of the geodesic which is minimal is a curve in $\Delta^{n}$ joining the given points of strictly shorter length than the minimal geodesic. As this is impossible, we deduce that corresponding minimal geodesics must have the same length, and therefore we have an isometry of $\partial \Delta^{n}$ with the corresponding complex in $\mathbb{R}^{n}$.

We now 'fill-in' the isometry across the interior of the tetrahedron. Repeating this process dimension by dimension gives the desired isometry between $\Delta^{n}$ and some region of $\mathbb{R}^{n}$.

Proof of Theorem B. The complex $K$ is a CAT(0) space by Theorem A, and by Lemma $4 \cdot 2$ we have that $\operatorname{Inv}_{p}(K)$ is a geodesic space. Therefore [BFW, proposition 6.2] applies, with the conclusion that every geodesic triangle in $K$ is flat, that is, isometric to its comparison triangle in $\mathbb{R}^{2}$. By Lemma $4 \cdot 3, K$ must be isometric to $\mathbb{R}^{n}$.

\section{REFERENCES}

[BBI] D. Burago, Y. Burago and S. Ivanov. A Course in Metric Geometry. Graduate Studies in Mathematics vol. 33 (American Mathematical Society, 2001). 
[BFW] S. M. Buckley, K. FAlK and D. J. Wraith. Ptolemaic spaces and CAT(0). Glasgow J. Math. 51 (2009), 301-314.

[BH] M. R. BRIDSON AND A. HAEFLIGER. Metric Spaces of Non-Positive Curvature (Springer-Verlag, 1999).

[BHX] S. M. BuCKleY, D. Herron AND X. XIE. Metric space inversions, quasihyperbolic distance and uniform spaces. Indiana J. Math. 57 (2008), 837-890.

[C] I. CHAVEL. Riemannian Geometry: a Modern Introduction (Cambridge University Press, 1993).

[FLS] T. FoERTSCH, A. LYTCHAK AND V. SChroeder. Nonpositive curvature and the Ptolemy inequality. Int. Math. Res. Not. IMRN (2007), article ID rnm100, 15 pages.

[K] D. C. KAY. Ptolemaic metric spaces and the characterization of geodesics by vanishing metric curvature. PhD thesis. Michigan State University (1963).

[S] I. J. Schoenberg. A remark on M. M. Day's characterization of inner-product spaces and a conjecture of L. M. Blumenthal. Proc. Amer. Math. Soc. 3 (1952), 961-964. 